\title{
Laser induced non-sequential double ionization investigated at and below the threshold for electron impact ionization
}

\author{
E Eremina, X Liu, H Rottke, W Sandner, A Dreischuh†, F \\ Lindner $\dagger$, F Grasbon†, G G Paulust, $\mathbf{H}$ Walther†, $\mathbf{R}$ \\ Moshammer $\ddagger$, B Feuerstein $\ddagger$ and J Ullrich $\ddagger$ \\ E-mail: rottke@mbi-berlin.de \\ Max-Born-Institut, Max-Born-Str. 2a, D-12489 Berlin, Germany \\ *Max-Planck-Institut für Quantenoptik, Hans-Kopfermann-Str. 1, D-85748 \\ Garching, Germany \\ + Max-Planck-Institut für Kernphysik, Saupfercheckweg 1, D-69117 Heidelberg, \\ Germany
}

\begin{abstract}
We use correlated electron-ion momentum measurement to investigate laser induced non-sequential double ionization of $\mathrm{Ar}$ and Ne. Light intensities are chosen in a regime at and below the threshold where, within the rescattering model, electron impact ionization of the singly charged ion core is expected to become energetically forbidden. Yet, we find $\mathrm{Ar}^{++}$ion momentum distributions and an electron-electron momentum correlation indicative of direct impact ionization. Within the quasistatic model this may be understood by assuming that the electric field of the light wave reduces the ionization potential of the singly charged ion core at the instant of scattering. The width of the projection of the ion momentum distribution onto an axis perpendicular to the light beam polarization vector is found to scale with the square root of the peak electric field strength in the light pulse. A scaling like this is not expected from the phase space available after electron impact ionization. It may indicate that the electric field at the instant of scattering is usually different from zero and determines the transverse momentum distribution. A comparison of our experimental results with several theoretical results is given.
\end{abstract}

PACS numbers: 33.80.Rv,33.80.Wz

Submitted to: J. Phys. B: At. Mol. Phys. 


\section{Introduction}

Double ionization of atoms in high intensity ultra-short laser pulses has been found to proceed in a non-sequential way as long as the probability for single ionization stays below unity. This first became evident in unusually high double ionization rates $[1,2,3,4,5]$. A first hint to the mechanism behind non-sequential double ionization (NSDI) came from the dependence of the total yield of doubly charged ions on the ellipticity of the light beam polarization $[4,6]$. The experiments indicated that inelastically scattered electrons that were driven back to the ion core by the oscillating laser field may be responsible for NSDI. The same electron scattering mechanism [7] is responsible for the generation of high-order harmonics of a strong ultra-short light pulse [8] and for the production of high-energy photoelectrons in single ionization [9]. Further confirmation of this mechanism comes from experiments where the momentum distributions of doubly charged He and Ne ions were measured $[10,11]$, from kinematicaly complete momentum spectroscopy on the final state electrons and on the ion $[12,13,14,15]$, and from electron kinetic energy distributions measured in coincidence with doubly charged ions $[16,17,18,19]$.

A classical analysis shows that the maximum kinetic energy $E_{k i n, \max }$ of the electron returning to the ion core is $3.17 U_{p}$, where $U_{p}$ denotes the ponderomotive energy. It is defined as the quiver energy of a free electron in an oscillating electric field. $U_{p}$ is proportional to the laser intensity $I$. Depending on $E_{k i n, \max }$ different possibilities for double ionization may be envisioned. First, instantaneous impact ionization of the singly charged ion. At first sight, for this to be possible, the kinetic energy $E_{k i n, \max }$ has to be larger than the ionization potential $I_{p}^{+}$of the ion core. Second, impact excitation of the ion core with subsequent electric field ionization of the excited ion in the light wave. For this to work, $E_{k i n, \max }$ has to be larger than the energy difference between the ion ground and first excited state. Experiments on Ar indicate that this mechanism may contribute to double ionization $[13,20]$. The assumption of this mechanism also seems to be necessary to reproduce theoretically the experimentally found ratio of double to single ionization yields for He [21]. Third, capture of the returning electron into an autoionizing state of the atom. The lifetime of such a state is usually long enough to facilitate electric field ionization of both excited electrons during the next cycles of the electric field of the light wave. Electric field ionization of a collision complex like this was discussed by Sacha and Eckhard [22].

The present knowledge of NSDI shows that inelastic electron-ion scattering is deeply involved in the mechanism. Highly differential experiments possible in strong field double ionization may therefore give deeper insight into electron-ion scattering, too.

Here we investigate a new regime of kinetic energies $E_{k i n, \max }$ for the electron returning to the ion core. $E_{k i n, \max }$ is close to or below the expected threshold for instantaneous impact ionization of $\mathrm{Ne}^{+}$and $\mathrm{Ar}^{+}$. For $E_{k i n, \max }<I_{p}^{+}$impact ionization should become impossible. Preceding experiments showed that the integral ion yield 
ratio $\left[A^{++}\right] /\left[A^{+}\right]$does not show any abrupt change on crossing this threshold $[3,23]$. Given the validity of the afore mentioned models for NSDI, this fact has remained a puzzle and may point to a gradual change of ionization mechanisms near threshold. We will show for Ar that, in fact, a gradual change is observed, although a significant amount of instantaneous impact ionization remains. Observation of impact ionization below threshold shows that the light wave actually influences the inelastic scattering event.

\section{Experimental setup}

The experimental technique used is based on correlated ion-electron momentum spectroscopy [24]. A description of the spectrometer setup can be found in $[11,25]$. In short, ions created in the focal spot of a laser beam at the point of intersection with a supersonic atomic beam are extracted by a small electric field $(1 \mathrm{~V} / \mathrm{cm}-7 \mathrm{~V} / \mathrm{cm})$. After passage of an acceleration and a field-free drift tube, the ions hit a position-sensitive microchannel-plate detector (diameter $80 \mathrm{~mm}$ ) equipped with a delay line anode for position encoding. The ion's time-of-flight is measured with a time-to-digital converter (resolution: $0.5 \mathrm{~ns}$ ). The measured time-of-flight of each ion together with the position where it strikes the detector allows the reconstruction of its complete initial momentum vector with a resolution of $\approx 0.1$ a.u. along both directions perpendicular to the atomic beam axis. The solid angle of detection is $4 \pi$.

Similar to the ions the electrons created in the focal spot are extracted by the same electric field into the opposite direction. After passage of a field free drift tube they hit a second position sensitive detector of the same type as used for ion detection. Again the time-of-flight of each electron hitting the detector and its impact position are measured by a second time-to-digital converter (resolution: $0.5 \mathrm{~ns}$ ). This allows the reconstruction of the momentum of the electron it had when it was born in the focal spot. To achieve a large solid angle of detection for electrons a homogeneous magnetic field (field strength up to about 20 Gauss) parallel to the extraction electric field is applied over the whole electron flight path. This allows a solid angle of detection of $4 \pi$ also for electrons up to a certain maximum transverse momentum which is determined by the magnetic field. We have chosen this momentum large enough to guide all relevant electrons to the detector. The electron momentum resolution is $\approx 0.02 \mathrm{a} . \mathrm{u}$..

Correlated ion-electron momentum spectroscopy does not allow one to use high target densities to avoid false coincidences. In our experiment they start to arise if more than one atom is in the focal spot while a laser pulse passes by. The reason for this is that we only measure the momentum of one doubly charged ion and of one electron coming with the ion. We therefore have no means to decide whether the electron and the doubly charged ion come from ionization of the same atom in the focal spot if more than one had been there. Only if also the momentum of a second electron is measured one could use momentum conservation to decide whether these three particles are correlated.

In the low light intensity range where we are investigating double ionization the 
only possibility to perform systematic measurements at a reasonable double ionization rate therefore is to use high laser pulse repetition rates and additionally admit more than one target atom to the focal spot if possible. A measure of the number of false coincidences can be derived from single ionization of target atoms. There momentum conservation can be employed. The sum-momentum component of the singly charged ion and of the corresponding photoelectron along the polarization vector of the light beam, which in our case is perpendicular to the atomic beam axis, has to be zero. False electron-ion coincidences violate momentum conservation. One thus expects a sum-momentum distribution consisting of a narrow line sitting on a broad background. This is what we observe. The ratio of the number of events in the broad background to the number of events in the narrow line is a measure for the ratio of false to true coincidences. In our case this ratio is always kept below $7 \%$.

We use a laser system with $100 \mathrm{kHz}$ repetition rate [26]. The pulse duration is $35 \mathrm{fs}$ FWHM and the pulse energy reaches up to $6 \mu \mathrm{J}$ at a central wavelength of $800 \mathrm{~nm}$. The laser beam is focused by a spherical mirror $(f=100 \mathrm{~mm})$ on the target atomic beam, which has a width of $\approx 50 \mu \mathrm{m}$ along the propagation direction of the light beam. This gives rise to an interaction volume of $\approx 2 \times 10^{-9} \mathrm{~cm}^{3}$. The highest light intensity reached in the focal spot is $\approx 250 \mathrm{TW} / \mathrm{cm}^{2}$. The crossed laser-atomic beam geometry results in a variation of the light intensity of at most $10 \%$ along the light beam propagation direction. The interaction volume together with a maximum target gas density of $5 \times 10^{9} \mathrm{~cm}^{-3}$ results in a number of $N \leq 10$ atoms in the interaction volume. The probability to ionize a target gas atom per laser shot is always kept below 0.1 . Under these conditions the above stated ratio of false to true coincidences is found. The background gas pressure is kept below $3 \times 10^{-10}$ mbar in experimental runs.

\section{Results and discussion}

\section{1. "Below" threshold impact ionization}

Fig. 1 displays $\mathrm{Ne}^{++}$and $\mathrm{Ar}^{++}$ion momentum distributions measured at several light intensities in a focused linearly polarized light beam. They are completely equivalent to sum-momentum distributions of the two photoelectrons leaving the atom. This correspondence is exact for observation parallel to the light beam polarization axis and very close to exact for observation parallel to the propagation direction of the beam (better than the available momentum resolution). The momentum distributions are arranged according to the light intensity. The relevant parameters are compiled in Table 1. The left panel of Fig. 1 shows $\mathrm{Ar}^{++}$(lines) and $\mathrm{Ne}^{++}$(squares connected by a line) momentum distributions projected onto the polarization axis of the light wave $f\left(p_{\|}\right)$. The right panel shows the corresponding distributions projected onto the light beam propagation axis $f\left(p_{\perp}\right)$. In Figs. 1a,b the kinetic energy of the recolliding electron is larger than the ionization threshold of the corresponding singly charged ion (see Table 1). It thus may kick out a second electron instantaneously upon recollision. 
On the other hand, in Figs. 1c,d the maximum kinetic energy of the returning electron is smaller than the ionization threshold of $\mathrm{Ar}^{+}$. At the lowest light intensity (Fig. 1d) it just suffices to excite $\mathrm{Ar}^{+}$from the ground state to the lowest lying bound excited states.

The functional form of the $\mathrm{Ne}^{++}$momentum distribution $f\left(p_{\|}\right)$does not significantly depend on the light intensity, even if it is reduced from far above $\left(E_{\text {kin,max }}-I_{p}\left(\mathrm{Ne}^{+}\right) \approx 200 \mathrm{eV}\right)$ to close to the threshold for instantaneous electron impact ionization (Fig. 1a, $E_{k i n, \max }-I_{p}\left(\mathrm{Ne}^{+}\right)=4.5 \mathrm{eV}$ ). This becomes obvious if one compares our present result (Fig. 1a) with the corresponding momentum distribution at $1.3 \mathrm{PW} / \mathrm{cm}^{2}$ in ref. [11]. It indicates that the main ionization mechanism at both light intensities and therefore certainly also in between is instantaneous electron impact ionization, preferentially near zero crossings of the oscillating electric field of the light wave [11]. On the other hand, the functional form of $f\left(p_{\|}\right)$for $\mathrm{Ar}^{++}$changes with decreasing kinetic energy of the returning electron (Figs. 1a,.., d and ref. [13]). It evolves from a double peaked structure at $E_{k i n, \max }-I_{p}\left(\mathrm{Ar}^{+}\right)=18 \mathrm{eV}$ to a bell shaped one with a single maximum at $p_{\|}=0$ at $E_{k i n, \max } \ll I_{p}\left(\mathrm{Ar}^{+}\right)$. The double hump structure which is characteristic of instantaneous impact ionization by a rescattering electron is less pronounced than for $\mathrm{Ne}$ and vanishes already above the expected threshold for impact ionization of $\mathrm{Ar}^{+}$(Fig. 1a,b). At first sight impact ionization thus seems to disappear with decreasing light intensity, already at $E_{k i n, \max }>I_{p}\left(\mathrm{Ar}^{+}\right)$.

A closer inspection of the electron sum-momentum distributions and of e-e correlation spectra for the momentum components of the two photoelectrons parallel to the light beam polarization vector on the other hand reveals that $\mathrm{e}^{-}$impact ionization of $\mathrm{Ar}^{+}$does not vanish with decreasing light intensity, not even if $E_{k i n, \max } \ll I_{p}\left(\mathrm{Ar}^{+}\right)$. Fig. 2 shows two such spectra taken at $150 \mathrm{TW} / \mathrm{cm}^{2}$ and $90 \mathrm{TW} / \mathrm{cm}^{2}$. The corresponding excess energies of the recolliding electron are given in Table 1. On the horizontal axis the momentum component $p_{1, \|}$ of the detected photoelectron is plotted and on the vertical axis the corresponding momentum component of the second electron $p_{2, \|}$. It is calculated from $p_{1, \|}$ and the measured momentum of $\mathrm{Ar}^{++}$using momentum conservation. At both light intensities two maxima along the main diagonal in the 1st and 3rd quadrants of the plots are found. They clearly indicate that instantaneous electron impact ionization of the $\mathrm{Ar}^{+}$ion core significantly contributes to double ionization even at $E_{\text {kin,max }} \ll I_{p}\left(\mathrm{Ar}^{+}\right)[12,13,14,15,20,31]$. The momentum correlation spectra show that electrons from instantaneous impact ionization always make up the sum-momentum distributions in Fig. 1 at large $\left|p_{\|}\right|$. They also reveal the reason for the disappearance of the double hump structure in the projected electron sum-momentum distributions in Fig. 1 with decreasing light intensity. An increasing amount of events with electrons emitted into opposite half spaces with significant and similar momenta appears in the 2nd and 4th quadrants. These events nearly completely make up the sum-momentum distribution near $p_{\|}=0$ at the lowest light intensity in Fig. 1d. A possible ionization mechanism which may give rise to these events was discussed in ref. [13].

Further evidence for instantaneous impact ionization below the threshold comes 
from the dependence of the "cutoff" of the sum-momentum distributions $f\left(p_{\|}\right)$on the light intensity. A "cutoff" momentum of $f\left(p_{\|}\right)$may be defined as the momentum where the yield of events in the spectra in Fig. 1 decreases to half of their maximum value. In Fig. 3 this "cutoff" is plotted for $\mathrm{Ar}$ and Ne versus the ponderomotive potential $U_{p}$. Squares represent data derived from Ar and dots data from Ne spectra. For the low $U_{p}$ data points the error in determining $U_{p}$ is similar to the respective symbol size. The data point at $U_{p}=2.8$ a.u. is taken from ref. [11]. The full line shows the function $4 \sqrt{U_{p}}$. It closely follows the data points, lying systematically slightly above. The slightly increasing deviation at small $U_{p}$ is caused by the events in the 2 nd and 4th quadrants of Fig 2 which are not formed by instantaneous impact ionization and determine the maximum yield at low light intensity in the sum-momentum distributions. They tend to decrease the "cutoff" momentum.

$4 \sqrt{U_{p}}$ is just the largest momentum the doubly charged ion, and therefore the electrons, can gain through post-collision acceleration in the light pulse [30]. The measured "cutoff" following this line indicates that the final sum-momentum component $p_{\|}$of the electrons is mainly determined by the action of the light pulse on the already free electrons. The data points smoothly following this line in crossing the ionization threshold of $\mathrm{Ar}^{+}$indicates that the double ionization mechanism which forms electrons with large $\left|p_{\|}\right|$remains instantaneous impact ionization. These electrons determine the "cutoff" of the bell shaped $f\left(p_{\|}\right)$in Fig. 1d. This is similar to what was found already at $E_{k i n, \max }>I_{p}\left(\mathrm{Ar}^{+}\right)[13]$.

In the quasistatic model instantaneous impact ionization below the expected threshold $E_{k i n, \max }=I_{p}^{+}$may be understood by taking into account that the electric field of the light wave usually is different from zero at the instant of time $t_{r}$ when the electron scatters. The actual threshold for electron impact ionization of a singly charged ion in an external electric field of strength $F \cos \omega t_{r}$ at the instant of scattering is therefore lowered to $I_{p}^{+}\left(t_{r}\right)=I_{p, 0}^{+}-2 \sqrt{2\left|F \cos \omega t_{r}\right|}$ (in atomic units), the instantaneous saddle point of the combined external and Coulomb potential. This relation is a good approximation of the real ionization threshold as long as the Stark shift of the ionic ground state remains small and the saddle point appears well outside of the electron charge cloud of the doubly charged ion core. Such a field dependent shift of the ionization threshold has already been introduced recently by van der Hart and Burnett in order to understand the missing threshold behavior in the dependence of the total ion yield ratio $\left[\mathrm{He}^{++}\right] /\left[\mathrm{He}^{+}\right]$on the light intensity for helium [32].

Fig. 4a shows the kinetic energy $E_{k i n}\left(t_{r}\right)$ of the returning electron (full line) and the instantaneous ionization potential $I_{p}^{+}\left(t_{r}\right)$ under the various conditions of our experiment. The energy is given in multiples of the ponderomotive energy $U_{p}$. The dash-dotted and dashed lines represent $I_{p}^{+}\left(t_{r}\right)$ at $240 \mathrm{TW} / \mathrm{cm}^{2}$ for $\mathrm{Ar}^{+}$and $\mathrm{Ne}^{+}$, respectively, while the dotted line is for $\mathrm{Ar}^{+}$at $90 \mathrm{TW} / \mathrm{cm}^{2}$. The electric field of the light wave crosses zero at $\omega t_{r} / \pi=1.5$. Electrons returning at this instant of time find the ionization threshold of the ion unperturbed; it therefore reaches a maximum. At all $t_{r}$ with $E_{k i n}\left(t_{r}\right) \geq I_{p}^{+}\left(t_{r}\right)$ the kinetic energy of the returning electron classically suffices to kick out a second 
electron instantaneously in an inelastic collision. Such an interval of return times is found for both atoms at all light intensities investigated. The $t_{r}$ dependent excess energy $E_{\text {exc }}$ after collision is plotted in Fig. $4 \mathrm{~b}$ in atomic units. It is given with respect to the saddle of the perturbed potential and is significantly different for Ar and Ne at the same light intensity $\left(240 \mathrm{TW} / \mathrm{cm}^{2}\right)$.

Theoretical results on non-sequential double ionization have already shown that instantaneous impact ionization "below" threshold should be observable [30]. This S-matrix calculation based on the quasistatic model, taking into account only instantaneous impact ionization by the recolliding electron, showed that electron summomentum distributions similar to those "above" threshold are expected.

Besides $f\left(p_{\|}\right)$, which is influenced by acceleration of the free photoelectrons (and of the ion) by the electric field of the light wave, $f\left(p_{\perp}\right)$ is expected to give insight into the recollision process. It is not affected by post-recollision acceleration but is determined in the recollision event while the colliding electron interacts with the singly charged ion core. The right panel of Fig. 1 shows $f\left(p_{\perp}\right)$ (the projection of $f(\vec{p})$ on the light beam propagation axis). As can be seen in Fig. 1a $f\left(p_{\perp}\right)$ for Ar and Ne are practically identical at the same light intensity $\left(240 \mathrm{TW} / \mathrm{cm}^{2}\right)$. This indicates that $f\left(p_{\perp}\right)$ is insensitive to the initial state from which the electrons are removed, for Ne from the (2p) and for $\mathrm{Ar}$ from the $(3 \mathrm{p})$ shell. Also, similar to $f\left(p_{\|}\right)$, the significantly differing excess energies of the electrons after recollision (see Table 1 ) do not affect $f\left(p_{\perp}\right)$.

The shape of $f\left(p_{\perp}\right)$ does not change with light intensity. Over the dynamic range of our experiment it can well be fitted with a Gaussian function at all light intensities. The insensitivity to the atomic species also shows up if the full width at half maximum (FWHM) of this function is plotted versus the square root of the peak electric field strength $\sqrt{E_{0}}$ of the light wave (Fig. 5). In Fig. 5 the FWHM for Ar is shown as squares and that for $\mathrm{Ne}$ as circles. All data points, except the one at $90 \mathrm{TW} / \mathrm{cm}^{2}$, are closely lying on a straight line through zero, i. e.: FWHM $\propto \sqrt{E_{0}}$. The high $E_{0}$ Ne data point, which is taken from ref. [11], shows that the corresponding large excess energy $E_{\text {kin, } \max }-I_{p}\left(\mathrm{Ne}^{+}\right) \approx 200 \mathrm{eV}$ which scales with $E_{0}^{2}$ does not seem to influence $f\left(p_{\perp}\right)$. Crossing of the threshold for impact ionization of $\mathrm{Ar}^{+}$at low $E_{0}$ initially also does not change the relation between the FWHM and $E_{0}$. A faster decrease in width is found only at the lowest light intensity $\left(90 \mathrm{TW} / \mathrm{cm}^{2}\right)$. Concerning this intensity one has to keep in mind that at $E_{k i n, \max } \ll I_{p}\left(\mathrm{Ar}^{+}\right)$a large amount of events not attributed to instantaneous impact ionization of $\mathrm{Ar}^{+}$contributes to $f\left(p_{\perp}\right)$ (see Fig. 2b). This may be responsible for the deviation.

The scaling of the FWHM of $f\left(p_{\perp}\right)$ with $\sqrt{E_{0}}$ is intriguing. One would expect a scaling with the available excess energy $E_{k i n, \max }-I_{p}^{+}$, that is, with $E_{0}$. It reminds one of the scaling found for the width of the momentum distribution of the photoelectron in strong field single ionization in the quasistatic limit (see for example [33]). The 
corresponding momentum distribution is [33]:

$$
f\left(\vec{p}_{\perp}\right)=w_{0} \exp \left[-\frac{\sqrt{2 I_{p}} \vec{p}_{\perp}^{2}}{E_{0}}\right]
$$

with $I_{p}$ the ionization potential of the atom. The FWHM of this Gaussian function is:

$$
\mathrm{FWHM}=\frac{2 \sqrt{\ln 2}}{\left(2 I_{p}\right)^{1 / 4}} \sqrt{E_{0}}
$$

Relation (1) for the transverse momentum distribution was derived in the tunneling regime where the electric field strength is small enough not to allow above barrier ionization. The width of this distribution depending on the electric field strength means that with increasing final transverse momentum the electron has tunnelled through a wider barrier away from the saddle point formed by the combined Coulomb and external electric field induced potential (see also ref. [34]).

Provided the similar behavior of $f\left(\vec{p}_{\perp}\right)$ for single ionization and for the electron sum-momentum distribution in double ionization is not accidental this means that at the instant of recollision the electric field of the light wave plays an important role to free the second electron. The returning electron may not transfer enough energy to the second one so that either the second electron or the whole collision complex gets ionized by tunnel or above barrier ionization in the presence of the external field [22]. The light electric field then would have a significant effect on the transverse momentum distribution of the two electrons, similar to single ionization. Barrier suppression or tunneling would favor small transverse momenta and a scaling of the width of $f\left(\vec{p}_{\perp}\right)$ with $\sqrt{E_{0}}$ as found in the experiment may result. This would mean that the collisions which most efficiently lead to double ionization do not happen at zero crossings of the electric field of the light wave and at the highest kinetic energy possible for the returning electron at $\omega t_{r} / \pi \leq 3 / 2$ (see Fig. $4 \mathrm{~b}$ ). It is presently not known whether such a scenario can reproduce the electron sum-momentum distribution parallel to the polarization axis of the light beam and the e- e momentum correlation found in the experiment.

In a one dimensional simulation of double ionization the recollision scenario outlined above was already discussed and found to be important by Eberly and coworkers [35], but its relevance in the full $3 \mathrm{~d}$ real world is presently not assured. In this simulation most efficient collisions happen at times $3 / 2<\omega t_{r} / \pi \leq 2$, where the recolliding electron is going to be stopped by the electric field of the light wave during or short after collision and returned back. As can be seen in Fig. 4b the available excess energy after collision in this time interval is small and steadily decreases to zero at $\omega t_{r} / \pi=2$. The recolliding electron in this situation is born by electric field ionization close to the maxima of the oscillating electric field $E(t)=E_{0} \cos \omega t$.

\subsection{Comparison to several theoretical results}

Fig. 1a shows that the electron sum-momentum distributions at the same light intensity $\left(240 \mathrm{TW} / \mathrm{cm}^{2}\right)$ measured for $\mathrm{Ar}$ and Ne virtually coincide at large $\left|p_{\|}\right|$down to 
$\left|p_{\|}\right| \approx 1.8$ a.u.. The "cutoff" momenta of the distributions are the same. This is further evidence that the same ionization mechanism, instantaneous impact ionization, gives rise to the large $\left|p_{\|}\right|$events for both atoms. The identical behavior of $f\left(p_{\|}\right)$at large $\left|p_{\|}\right|$ is remarkable in view of the fact that the excess energies $E_{\text {exc }}$ after inelastic scattering of the returning electron differ significantly (Fig. 4b). The larger $E_{\text {exc }}$ available for Ar does not give rise to electrons with larger sum-momentum $\left|p_{\|}\right|$. Furthermore, the two maxima of $f\left(p_{\|}\right)$for Ar appear at a slightly smaller sum-momentum than for Ne. The larger accessible phase space for Ar would suggest to find a larger "cutoff" and position for the maxima of $f\left(p_{\|}\right)$. In fact, model calculations showed this difference [30]: $f\left(p_{\|}\right)$ was calculated for $\mathrm{Ar}$ and $\mathrm{Ne}$ at $U_{p}=11.9 \mathrm{eV}$ close to $U_{p}=14.3 \mathrm{eV}$ we have in the experiment. The strong field S-matrix approach with an e-e contact interaction was used in the calculation. The broader $f\left(p_{\|}\right)$found theoretically for Ar nearly exactly corresponds to the extended accessible phase space compared to Ne. Since the contact interaction for scattering does not prefer certain momenta of the electrons directly after scattering it is not surprising that the correspondingly different accessible phase space volumes for $\mathrm{Ar}$ and $\mathrm{Ne}$ at the same light intensity give rise to a difference in the summomentum distributions. The different experimental finding indicates that the contact interaction is an oversimplification. It is not able to reproduce the insensitivity of the electron sum-momentum distributions to the excess energy after impact ionization.

An S-matrix approach to calculate electron sum-momentum distributions was also used in ref. [36], incorporating the correct Coulomb e-e interaction. In ref. [36] a "cutoff" formula for $f\left(p_{\|}\right)$was derived to be $p_{\|, \text {cutof } f}=R e\left(4 \sqrt{U_{p}}+2 \sqrt{2 U_{p}-I_{p}^{+}}\right)$. The "cutoff" definition differs from the experimental one but at low light intensity its value is identical to the $4 \sqrt{U_{p}}$ found in the experiment and equal to the largest momentum which can be gained by acceleration in the light pulse. But an increasing deviation from the experiment is found for $2 U_{p}>I_{p}^{+}$where the second square root in the formula starts to influence the "cutoff". For the high intensity Ne data point in Fig. 1 it gives a "cutoff" momentum of 10.4 a.u.. This is significantly larger than the measured "cutoff" for this atom using our definition of the "cutoff" above.

Aside from the quantitative difference to the experiment the S-matrix approach with the contact interaction reproduces the experimental finding that at all light intensities investigated so far the electrons from instantaneous impact ionization are emitted preferentially with similar momentum components parallel to the light beam polarization vector (highest probability to find electron pairs along the main diagonal in Fig. 2; see also refs. $[12,13,15])$. This behavior is not found if the contact e-e interaction in the S-matrix is replaced by the more realistic Coulomb interaction and the 1st order Born approximation is used. It favors different electron momentum components along the polarization vector in the final state, especially with increasing light intensity [31, 37].

The correct Coulomb e-e interaction was also used in a semiclassical trajectory calculation to model non-sequential double ionization [38]. It was based on the rescattering model. But, in addition to e-e repulsion to all orders also the attractive Coulomb interaction of both electrons with the ion core was included exactly. With 
this done the momentum correlation $f\left(p_{1, \|}, p_{2, \|}\right)$ found is qualitatively similar to that arrived at experimentally and theoretically with the e-e contact interaction (see above) [31]. Both electrons tend to have similar $\left(p_{1, \|}, p_{2, \|}\right)$, the probability distribution peaks at the main diagonal of the momentum correlation plot as the experimental one does (see Fig. 2). It also shows two separate maxima along this line displaced from $\left(p_{1, \|}, p_{2, \|}\right)=(0,0)$. This may indicate that it is necessary to incorporate into a quantum mechanical calculation besides the e-e Coulomb interaction also the e-ion core interaction. It seems to suppress the tendency to find unequal electron momentum components $p_{1, \|}, p_{2, \|}$ for the electrons directly after scattering and therefore in the final momentum correlation function $f\left(p_{1, \|}, p_{2, \|}\right)[31,37]$.

\section{Conclusions}

In conclusion, we presented experimental data at light intensities where impact ionization by the returning electron is classically forbidden at first sight. Nevertheless, our data show ion momentum distributions and an e-e momentum correlation which are characteristic of instantaneous impact ionization. Within the quasistatic model this can be explained via a lowering of the unperturbed ionic ionization potential by the electric field of the light wave present at the instant of scattering. The electric field thus essentially influences the scattering event. The dependence on light intensity of $f\left(p_{\perp}\right)$ seems to show that this is also the case for $E_{k i n, \max } \gg I_{p}^{+}$. An alternative way to understand the observed impact ionization "below" threshold is to assume that the collision complex which forms when the electron returns to the ion core absorbs photons. If a sufficient number is absorbed the internal energy suffices for instantaneous double ionization of the atom.

The comparison of our experimental with several theoretical results shows that specific discrepancies exist. They most obviously show up in qualitative differences of electron momentum correlation spectra and, what is less surprising in view of the approximations which were made, in quantitative discrepancies. The dependence of the width of the transverse sum-momentum distribution on light intensity may indicate that the electric field at the time of recollision always plays a decisive role during collision even at high light intensity. Comparing Ar to Ne data atom specific features appear. For Ne from far above the second ionization threshold to close to it instantaneous electron impact ionization of $\mathrm{Ne}^{+}$prevails. For Ar already for $E_{k i n, \max }>I_{p}\left(\mathrm{Ar}^{+}\right)$a second ionization mechanism starts to compete with instantaneous impact ionization.

\section{Acknowledgments}

We gratefully acknowledge support by the Deutsche Forschungsgemeinschaft (DFG). We thank W. Becker for fruitful discussions. 


\section{References}

[1] l'Huillier A, Lompre L A, Mainfray G and Manus C 1983 Phys. Rev. A 272503

[2] Fittinghoff D N, Bolton P R, Chang B and Kulander K C 1992 Phys. Rev. Lett. 692642

[3] Walker B et al. 1994 Phys. Rev. Lett. 731227

[4] Fittinghoff D N, Bolton P R, Chang B and Kulander K C 1994 Phys. Rev. A 492174

[5] Augst S et al. 1995 Phys. Rev. A 52 R917

[6] Dietrich P, Burnett N H, Ivanov M and Corkum P B 1994 Phys. Rev. A 50 R3585

[7] Corkum P B 1993 Phys. Rev. Lett. 711994

[8] Ferray M et al. 1988 J. Phys. B: At. Mol. Opt. Phys. 21 L31

[9] Paulus G G, Nicklich W, Xu Huale, Lambropoulos P and Walther H 1994 Phys. Rev. Lett. 72 2851

[10] Weber Th et al. 2000 Phys. Rev. Lett. 84443

[11] Moshammer R et al. 2000 Phys. Rev. Lett. 84447

[12] Weber Th et al. 2000 Nature 405658

[13] Feuerstein B et al. 2001 Phys. Rev. Lett. 87043003

[14] Moshammer R et al. 2002 Phys. Rev. A 65035401

[15] Moshammer R et al. 2003 J. Phys. B: At. Mol. Opt. Phys. 36 L113

[16] Witzel B, Papadogiannis N A and Charalambidis D 2000 Phys. Rev. Lett. 852268

[17] Lafon R et al. 2001 Phys. Rev. Lett. 862762

[18] Peterson E R and Bucksbaum P H 2001 Phys. Rev. A 64053405

[19] Chaloupka J L, Rudati J, Lafon R, Agostini P, Kulander K C and DiMauro L F 2003 Phys. Rev. Lett. 90033002

[20] Weckenbrock M et al. 2001 J. Phys. B: At. Mol. Opt. Phys. 34 L449

[21] Bhardwaj V R et al. 2001 Phys. Rev. Lett. 863522

[22] Sacha K and Eckhardt B 2001 Phys. Rev. A 63043414

[23] Sheehy B et al. 1998 Phys. Rev. A 583942

[24] Ullrich J et al. 1997 J. Phys. B: At. Mol. Opt. Phys. 302917

[25] Moshammer R et al. 2001 Optics Express 8358

[26] Lindner F et al., submitted

[27] The light intensity was determined by two methods with consistent results. The $\mathrm{Kr}^{+}$ion yield was determined as a function of the light intensity and compared to ref. [28]. Photoelectron spectra for single ionization of Ar were taken and compared to precision measurements in ref. [29]. The values given in the text are accurate to within $\pm 15 \%$.

[28] Talebpour A et al. 1997 J. Phys. B: At. Mol. Opt. Phys. 301721

[29] Paulus G G et al. 2001 Phys. Rev. A 64021401

[30] Kopold R, Becker W, Rottke H and Sandner W 2000 Phys. Rev. Lett. 853781

[31] Goreslavskii S P, and Popruzhenko S V 2001 Optics Express 8 395; Goreslavskii S P, Popruzhenko S V, Kopold R and Becker W 2001 Phys. Rev. A 64053402

[32] van der Hart H W and Burnett K 2000 Phys. Rev. A 62013407

[33] Delone N B and Krainov V P 1991 J. Opt. Soc. Am. B 81207

[34] Gottlieb B, Lohr A, Becker W and Kleber M 1996 Phys. Rev. A 54 R1022

[35] Panfili R, Haan S L and Eberly J H 2002 Phys. Rev. Lett. 89113001

[36] Becker A and Faisal F H M 2000 Phys. Rev. Lett. 843546

[37] Becker A and Faisal F H M 2002 Phys. Rev. Lett. 89193003

[38] Fu Li-Bin, Liu Jie and Chen Shi-Gang 2002 Phys. Rev. A 65021406 
Table 1. Summary of parameters relevant for the experiment: the light intensity $I$ [27], the corresponding ponderomotive potential $U_{p}=I /\left(4 \omega^{2}\right)$ (in a. u.), the maximum kinetic energy of the returning electron $E_{k i n, \max }=3.17 U_{p}$, and the maximum excess energy with respect to the ionization potentials of $\mathrm{Ar}^{+}$and $\mathrm{Ne}^{+}$. For Ne the data are given only at that light intensity where we made an experiment.

\begin{tabular}{c|c|c|c|c}
$I\left[\mathrm{TW} / \mathrm{cm}^{2}\right]$ & $U_{p}[\mathrm{eV}]$ & $E_{\text {kin }, \max }[\mathrm{eV}]$ & $E_{\text {kin, } \max }-I_{p}\left[\mathrm{Ar}^{+}\right][\mathrm{eV}]$ & $E_{k i n, \max }-I_{p}\left[\mathrm{Ne}^{+}\right][\mathrm{eV}]$ \\
\hline 240 & 14.3 & 45.5 & 18 & 4.5 \\
150 & 9.3 & 29.5 & 1.9 & - \\
110 & 6.5 & 20.5 & -7.2 & - \\
90 & 5.4 & 17.2 & -10.5 & -
\end{tabular}



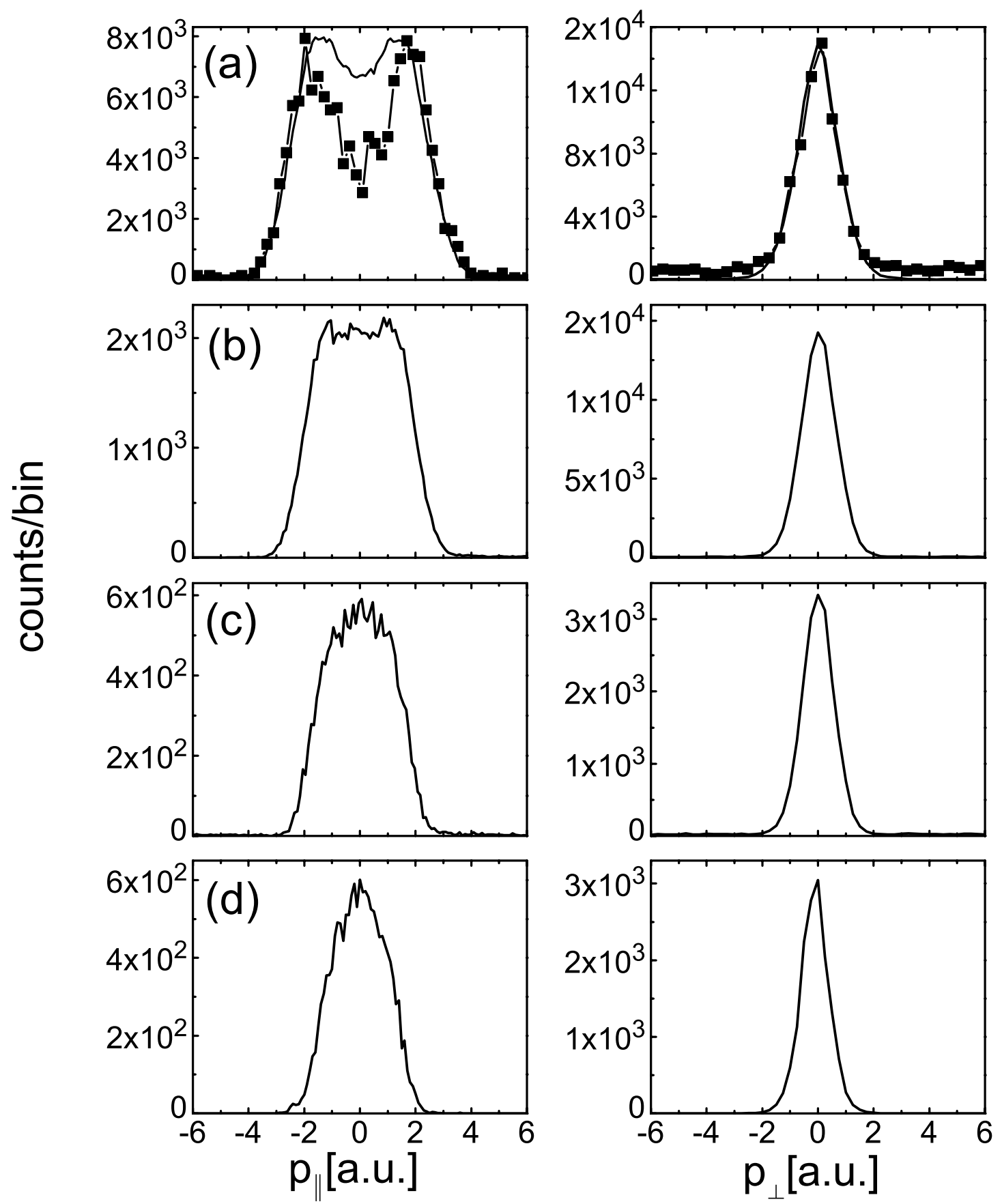

Figure 1. $\mathrm{Ar}^{++}$and $\mathrm{Ne}^{++}$momentum distributions parallel (left panel) and perpendicular (right panel) to the polarization vector of the light wave. (a) Ar (line) and $\mathrm{Ne}$ (connected squares) data at $240 \mathrm{TW} / \mathrm{cm}^{2}$; (b)...(d) $\mathrm{Ar}$ at decreasing light intensity: (b) $150 \mathrm{TW} / \mathrm{cm}^{2}$, (c) $110 \mathrm{TW} / \mathrm{cm}^{2}$, and (d) $90 \mathrm{TW} / \mathrm{cm}^{2}$. For (a) and (b) the maximum kinetic energy of the recolliding electron is larger than the ionization threshold $I_{p}^{+}$of the respective singly charged ion while for (c) and (d) it is lower. Further details are given in the text and in Table 1. 

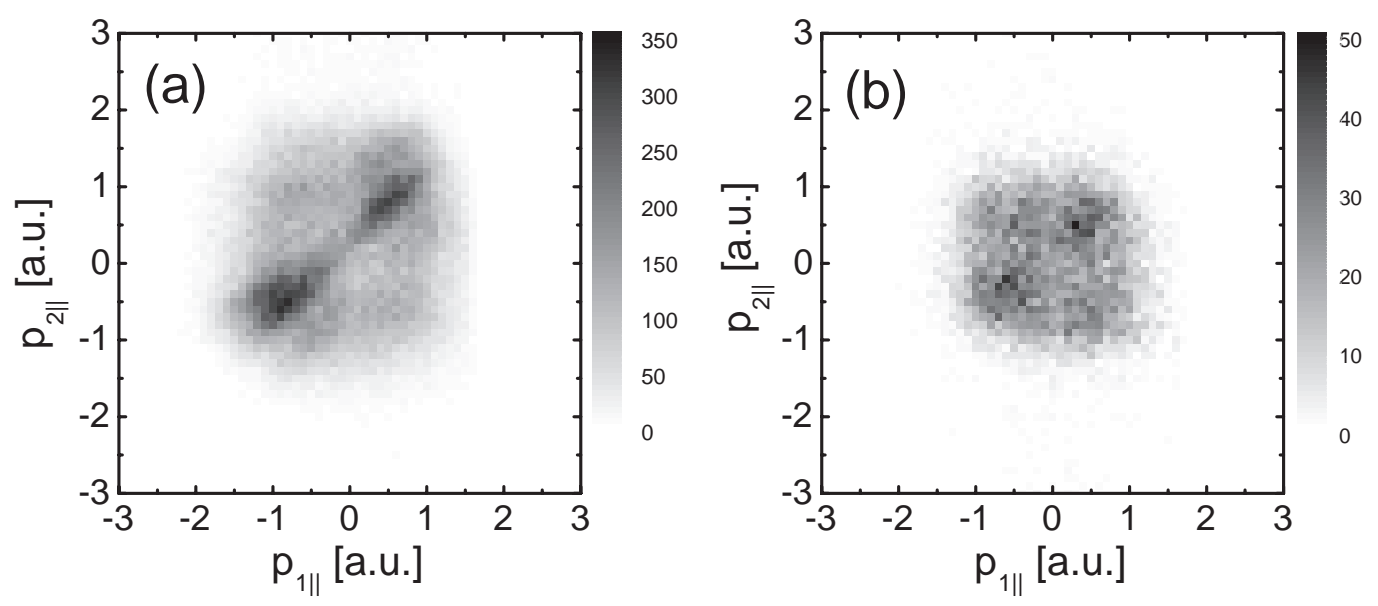

Figure 2. The momentum correlation of the two photoelectrons after double ionization of Ar. The momentum components along the light beam polarization axis are shown: (a) at $150 \mathrm{TW} / \mathrm{cm}^{2}$ and (b) at $90 \mathrm{TW} / \mathrm{cm}^{2}$. For (a) the maximum kinetic energy of the recolliding electron is larger than the ionization threshold $I_{p}\left(\mathrm{Ar}^{+}\right)$while for (b) it is well below the threshold. 


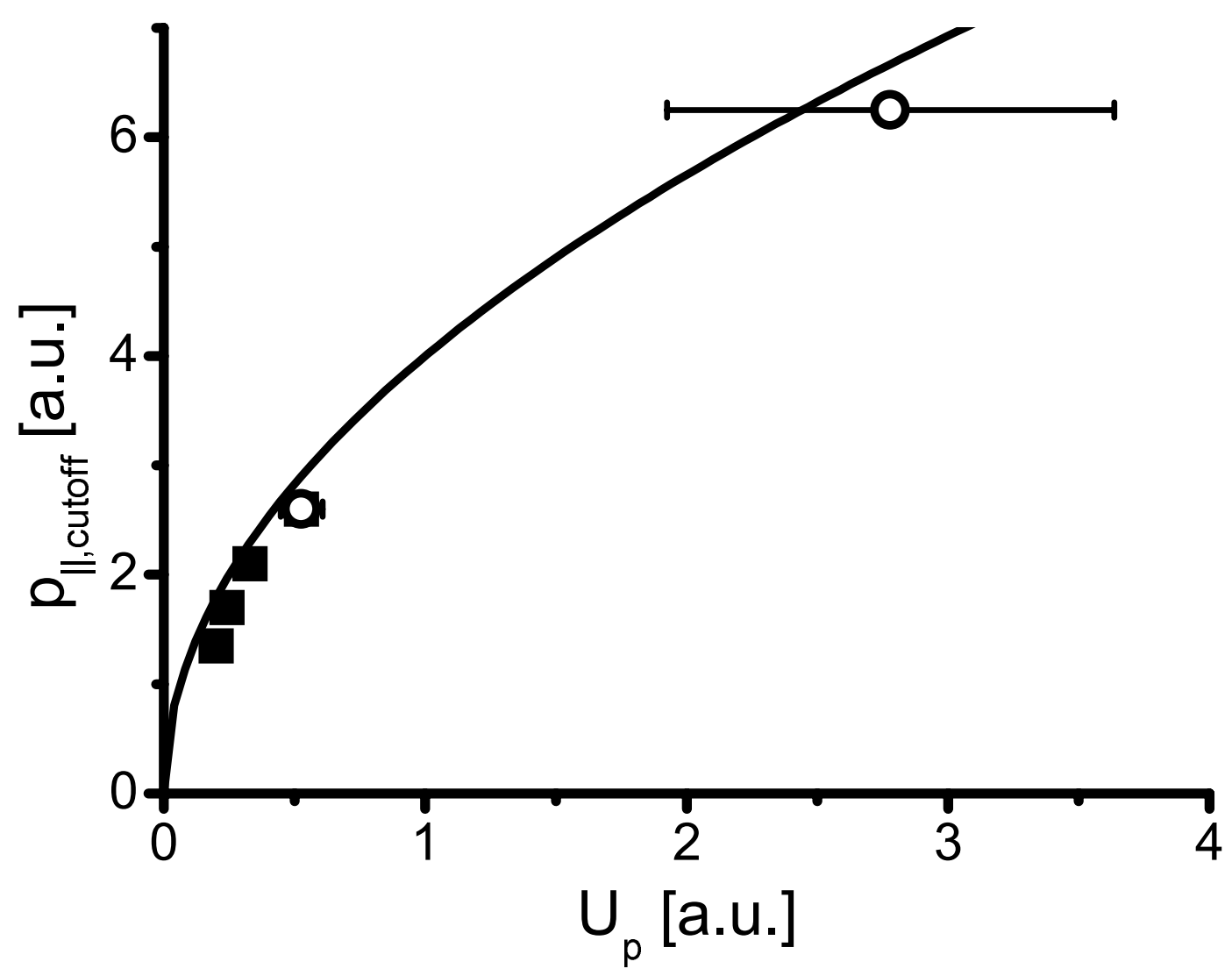

Figure 3. The dependence of the "cutoff" of the doubly charged ion momentum distributions $f\left(p_{\|}\right)$on the light intensity determined for $\mathrm{Ar}^{++}$(squares) and $\mathrm{Ne}^{++}$ (open circles). The line gives the calculated maximum momentum $\left(4 \sqrt{U_{p}}\right)$ which can be imparted on the ion by the light pulse. 


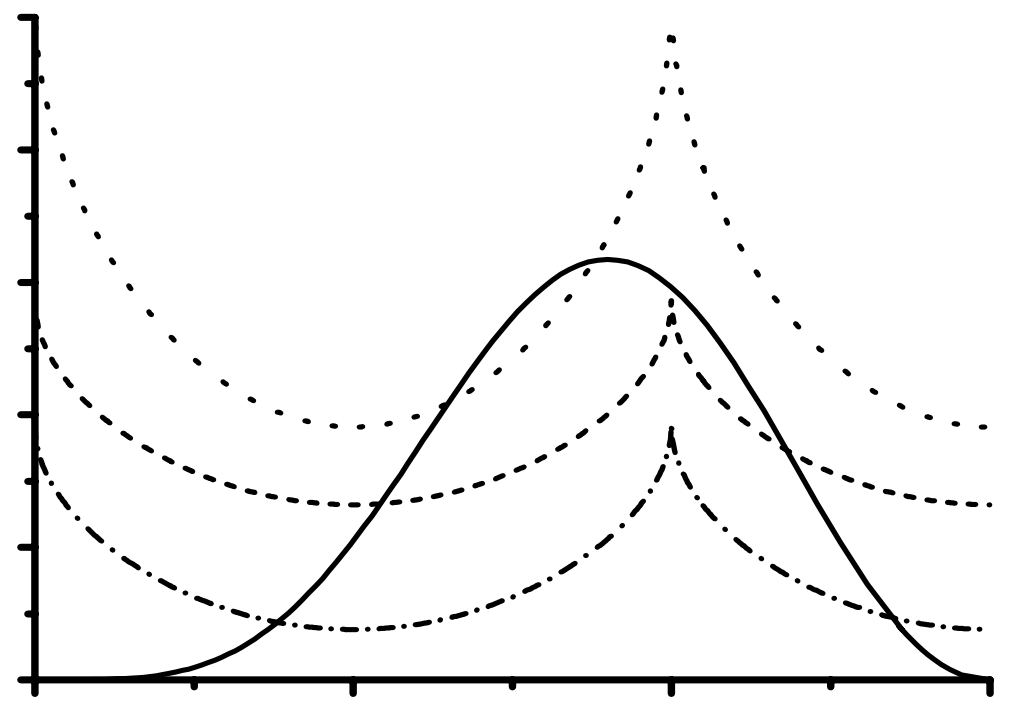

$\omega \pi$

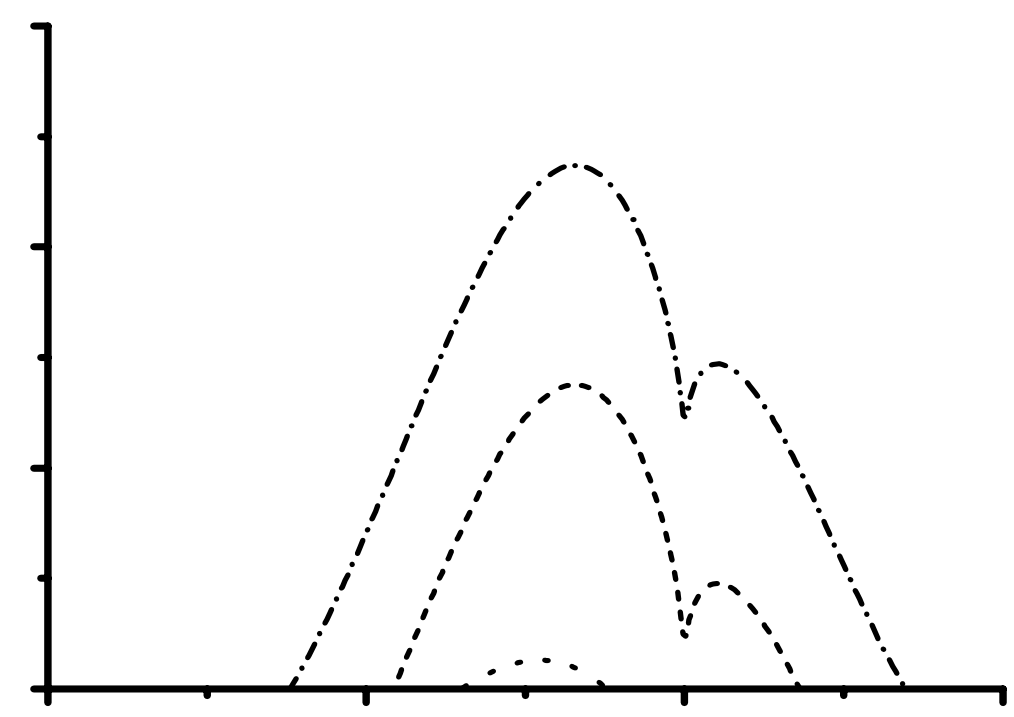

$\omega \pi$

Figure 4. (a) The instantaneous ionization potential of $\mathrm{Ar}^{+}$and $\mathrm{Ne}^{+}$at $t_{r}$, the time of recollision: - - - $\mathrm{Ar}^{+}, 240 \mathrm{TW} / \mathrm{cm}^{2} ; \cdots \mathrm{Ar}^{+}, 90 \mathrm{TW} / \mathrm{cm}^{2} ;---\mathrm{Ne}^{+}, 240 \mathrm{TW} / \mathrm{cm}^{2}$. Full line: the kinetic energy of the returning electron. (b) The corresponding excess kinetic energy $E_{\text {exc }}\left(t_{r}\right)=E_{k i n}\left(t_{r}\right)-I_{p}^{+}\left(t_{r}\right)$ after impact ionization. 


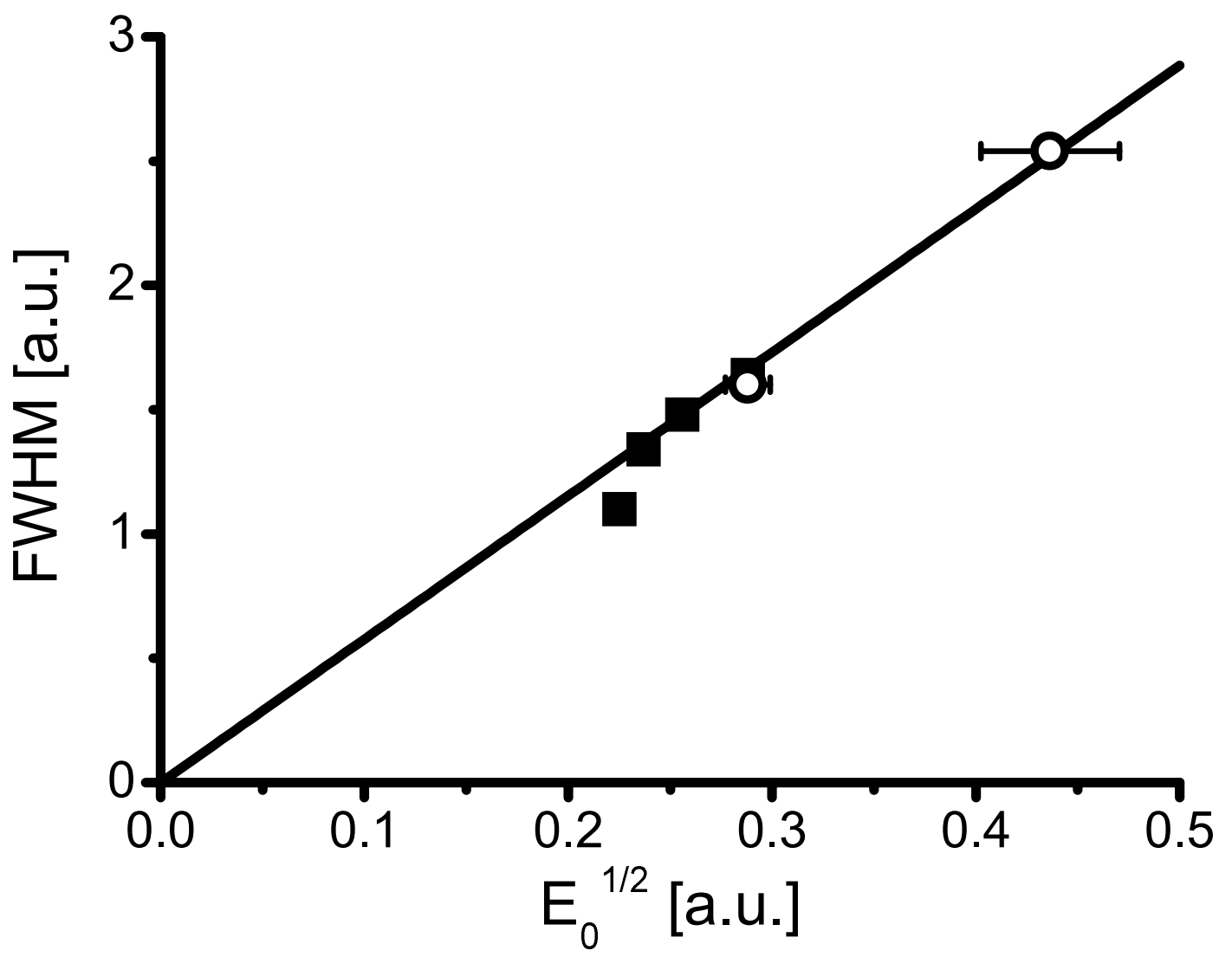

Figure 5. The FWHM of the doubly charged ion momentum distributions $f\left(p_{\perp}\right)$ plotted versus $\sqrt{E_{0}}$ (with $E_{0}$ the peak electric field strength). Squares are data derived from Ar and circles Ne data, respectively. The straight line visualizes the linear relation between the FWHM and $\sqrt{E_{0}}$ for the high intensity data points. 